\title{
Emotional Support for Men and Women With Cancer: Do Patients Receive What Their Partners Provide?
}

\author{
Aleksandra Luszczynska, Sonja Boehmer, Nina Knoll, Ute Schulz, \\ and Ralf Schwarzer
}

\begin{abstract}
Objectives: In the context of mainly gastrointestinal cancer surgery, the study examines the course of spousal support in 173 dyads over half a year to illuminate the function of gender in support transactions. Method: Provided and received emotional support were assessed in 108 male patientlfemale partner couples and 65 female patient/male partner couples. Using the Berlin Social Support Scales, assessments took place during the week before cancer surgery, 1 month, and 6 months after cancer surgery. Results: Gender differences emerged for support received and provided. Support received from partners was initially high for all patients, remained high over time for men, but decreased for women. Provided support decreased for male partners, but remained high in female partners. The effects were of medium size. Patients' received support was reflected by partners' reports of support provided. Women who reported received support 6 months after surgery had partners who had reported support provision 5 months earlier. Conclusions: Alternative sources of support, in particular for women, such as their network of friends or professional help, may need to be identified. A couple-coping intervention could be implemented to help partners learn about each other's needs in times of crisis and ways to cope with adversity.
\end{abstract}

Key words: social support, spouses, cancer, coping, couples, dyadic coping

Diagnosis and treatment of cancer are challenging to both patients and their partners. An acute medical situation can turn into a chronic condition when treatment is not successful or when side effects of surgery and therapy lead to severe life constraints. Patients may live with a constant threat of recurrence, experience compromised quality of life, and undergo vocational, emotional, and social changes. In these times of need, partners play an important role in patients'

Aleksandra Luszczynska,University of Sussex, England, and Warsaw School of Social Psychology, Poland; Sonja Boehmer, University of Sussex, England; Nina Knoll, Charité Universitätsmedizin Berlin, Germany; Ute Schulz, German Scholars Organisation, Berkeley, CA, USA; Ralf Schwarzer, Freie Universität Berlin, Germany.

The authors are indebted to the following cooperation partners in four Berlin hospitals and tumor treatment centers for their support of the study: Rotraut Asche-Messerschmidt, Bartholomäus Böhm, Konstanze Bössenrodt, Heinz-Johannes Buhr, Christoph-Thomas Germer, Alfred Holzgreve, Tido Junghans, Martina Lang, Joachim Müller, and Peter Neuhaus. The authors are also grateful to Nihal E. Mohamed, Charis Förster, and Steffen Taubert of the Coping with Surgery Research Team at the Freie Universität Berlin. Aleksandra Luszczynska was an Alexander von Humboldt Fellow. Sonja Boehmer and Ute Schulz have been awarded a Feodor Lynen Research Fellowship.

Correspondence concerning this article should be addressed to Ralf Schwarzer, Gesundheitspsychologie 10, Freie Universität Berlin, Habelschwerdter Allee 45, 14195 Berlin, Germany. E-mail: health@zedat.fu-berlin.de adjustment to illness (DeLongis et al., 2004; Revenson et al., 2005). Support from a partner has been shown to influence how patients adjust to their disease (Bodenmann, 1997; Clark \& Stephens. 1996; Coyne \& Fiske, 1992; Coyne \& Smith, 1991). Several studies have documented that emotional spousal support is associated with adaptation to and recovery from cancer (Helgeson, 1993; Revenson, 1994), immune parameters (Kiecolt-Glaser et al., 2002), and positive mood (Manne et al., 1999). Greater quality of support was associated with healthier neuroendocrine functioning in breast cancer patients (Turner-Cobb et al., 2000). However, it is possible that support is not always present, and, even if it is, it may be provided inconsistently over time. For example, in both patients and spouses, support has been found to decrease over the course of a year (see also Moyer \& Salovey, 1999; Northouse et al., 2000).

\section{Received Emotional Support}

Several types of social support have been investigated, such as instrumental (e.g., assist with a problem, donate goods), informational (e.g., give advice), and emotional support (e.g., give reassurance). Social support operates best when matched to the particular situation at hand (Burleson, 2003; Schwarzer, 
et al., 1994; Schwarzer, et al., 2004). Emotional support may be beneficial for recovery from surgery because it can instill optimistic self-beliefs and encourage the patient to cope with discomfort and relapses (Schulz \& Schwarzer, 2004). Emotional support seems to be more relevant than any other type of support. A previous study (Schulz \& Schwarzer, 2004), designed to examine support effects on coping, found similar levels and changes in received emotional support for 70 male and 38 female patients, as well as a beneficial effect of emotional support on subsequent coping strategies. Women also have been found to provide more emotional support than men (e.g., Cutrona, 1996; MacGeorge et al., 2003). Our study focuses on selfreported emotional support received by patients and provided by their intimate partners.

\section{Reports of Provided and Received Support}

It is assumed that partners' reports of support provided are to some degree reflected in patients' reports of support received. One would not expect, however, an accurate match between the level of support provision reported by the partner and the amount of received support reported by the patient. Support providers might misperceive the amount of support they extend, in line with a self-serving bias, seeing themselves as empathetic and caring, whereas recipients might harbor a different impression of the provider's behavior or intentions. In a study by Coriell and Cohen (1995), there was only moderate agreement within dyads about the occurrence of supportive behaviors. Dyad intimacy was associated with greater concordance.

Partners may also try to protect patients by buffering bad news or negative events, thus shielding the patient from adverse circumstances (Coyne \& Fiske, 1992; Coyne \& Smith, 1991). In a similar vein, "invisible support" is considered important, for example, when partners provide instrumental support without letting patients know. Patients cannot report this type of support because they are unaware of it (Bolger et al., 2000). Patients may also misperceive or under-report the amount of support they believe they receive. Or they may even not realize that they need support. Negative affect, such as depression, could cloud the perception of helpful acts or undermine beliefs about how much others care (Cutrona et al., 1997; Manne et al., 1999). In sum, a moderate association between partner's report of support provided and patient's report of support received can be expected.

\section{Gender Differences in Received and Provided Social Support}

Gender differences in social support have been discussed by various authors (cf. Glynn et al., 1999; Goldsmith \& Dun, 1997; Neff \& Karney, 2005). The question of how much support is received and how much is provided by men and women is a controversial topic (Gurung et al., 2003). Women provide more emotional support to both men and women, and, on average, they seem to get more help in return (Klauer \& Winkeler, 2002). Explanations for such discrepancies focus on gender differences in emotionality and emotional expressiveness (Burleson, 2003). Women generally have more close friends than men, and they tend to emphasize intimacy, empathy, and self-disclosure in their friendships. In short, women seem to devote more of themselves to their family and friends than men do, which is why they may often receive more support in return (Cutrona, 1996; Glynn et al., 1999). In spite of evidence on gender differences in received support in general, its temporal course is unknown-that is, whether differences in support received or provided narrow or widen over time.

\section{Aim of the Study}

This study examines emotional support provided by intimate partners and received by men and women with cancer using repeated measures over a period of six months. The distinction between gender and role is designed to clarify the function of gender in support transactions. It will be examined in which kind of dyad the amount of received support is higher. For example, male patients could receive more support because women are expected to be better support providers than men, an assumption that suggests an asymmetry of support transactions within couples. We have posed the following hypotheses.

First, levels of support are expected to be initially equally high for both men and women because at the onset of a major life event the situational demands override individual differences. Men would receive high emotional spousal support continuously over the halfyear period, whereas women would experience a decline in received support, based on the assumption that, within couples, men normally benefit more emotionally than women.

Second, due to the suggestion that women are more dependable caregivers, female partners are expected to provide emotional support in a more stable manner than male partners.

Third, the relationship between support provided and support received is expected to be gender-specific. Associations might differ between male patient/female partner dyads and female patient/male partner dyads. Directions of the effects are to be explored.

The fourth hypothesis refers to the dyad-specific prediction of received support. In female patient/male partner dyads there might be more substantial effects. It might be more relevant for women to be given emotional attention, whereas men might take it for granted to be cared for. 


\section{Method}

\section{Procedure}

The sample was recruited from the Berlin Longitudinal Study of Quality of Life after Tumor Surgery. After approval by the ethics committee, data were collected in cooperation with four hospitals in Berlin, Germany, in 2003. Research assistants interviewed patients in the week before surgery (Time 1) and asked them to fill in a questionnaire and an informed consent statement. Inclusion criteria were (a) preliminary diagnosis of a cancer that affects both men and women, (b) surgery scheduled within the next three days, and (c) fluency in German. Patients were later invited to participate in the follow-ups by sending them a questionnaire one month (Time 2) and six months post surgery (Time 3).

Partners were recruited through the patients. During the first wave of data collection, patients were asked by the interviewers whom they considered their closest person. At the end of the interview, a partner questionnaire and a stamped and self-addressed envelope were supplied. Patients were instructed to give this partner questionnaire to their significant other whom they had just identified during the interview. Partners received their questionnaire before surgery and one month after surgery.

\section{Participants}

Four hundred and eighty cancer patients, $60.2 \%$ men; mean age $=62, S D=11.8$, were recruited about three days before their surgery. One month following surgery, 294 patients $(61 \%)$ remained in the study, and six months following surgery, 233 patients (79\%) continued to participate. Attrition bias was examined by comparing patients who participated in all assessments with those who did not, taking into consideration all medical (e.g., type of surgery, comorbidity) and demographic (e.g., age, gender) variables. No significant differences between the two groups were found.

The analysis was based on patients whose intimate partner had completed the questionnaire at two time points (before surgery and one month later). Of 224 significant others, $173(77 \%)$ were intimate partners (spouses or equivalent opposite-sex couples), and the remaining 51 were children, grandchildren, siblings, parents, or friends. For the present analysis, only partners providing support who were in intimate relationships with the patient (e.g., husband, wife, or equivalent) were included. Children, friends, and other persons serving as caregivers were excluded.

Thus, a total of 173 couples were included in the study, 108 male and 65 female patients and their intimate partners. The mean age of patients was 63 years, $S D=9.5$, for men, and 60 years, $S D=12.2$, for women. On average, male partners were $60, S D=12$, and fe-
Table 1. Patient Sample Characteristics by Gender in Terms of Illness Attributes

\begin{tabular}{lcc}
\hline & $\begin{array}{c}\text { \% Male patients } \\
\text { \% }(\boldsymbol{n}=\mathbf{1 0 8})\end{array}$ & $\begin{array}{c}\text { \% Female patients } \\
\boldsymbol{\%}(\boldsymbol{n}=\mathbf{6 5})\end{array}$ \\
\hline Site of cancer & & \\
Colon & 22.6 & 21.9 \\
Rectum & 24.5 & 26.6 \\
Stomach & 12.3 & 7.8 \\
Esophagus & 4.7 & 3.1 \\
Pancreas & 3.8 & 10.9 \\
Liver/gall bladder & 10.4 & 7.8 \\
Lung/bronchi & 7.5 & 4.7 \\
Other & 14.1 & 17.2 \\
Time elapsed between diagnosis and Time & \\
<1 month & 58.6 & 57.8 \\
1-3 months & 21.8 & 22.2 \\
> 3 months & 19.5 & 20.0 \\
Surgical therapy & & \\
Curative & 89.4 & 91.1 \\
Palliative & 10.6 & 8.9 \\
Cancer stage & & \\
Stage I & 22.5 & 23.1 \\
Stage II & 22.5 & 19.2 \\
Stage III & 27.5 & 38.5 \\
Stage IV & 27.5 & 19.2 \\
\hline
\end{tabular}

male partners were 58.5 years old, $S D=11$. Illness attributes (type of cancer, stage of disease, received treatment) were unrelated to patients' gender and age. Table 1 provides an overview of all available disease characteristics for men and women.

\section{Measures}

The Berlin Social Support Scales (BSSS; Schulz \& Schwarzer, 2003) were used to assess various dimensions of social support. Received emotional support was measured by instructing patients to think about the person who is closest to them, such as their spouse. This instruction was followed by six items assessing emotional support (translated from German): (1) "This person showed me that he/she loves and accepts me," (2) "This person comforted me when I was feeling bad," (3) "This person made me feel valued and important," (4) "This person expressed concern about my condition," (5) "This person assured me that I can rely completely on him/her," and (6) "This person encouraged me not to give up"; Cronbach's alpha $=.85$. Items of provided support were intended to be parallel to the received support items and were measured by instructing partners, "Now think about the patient. How did you interact with him/her during the past week?" In line with the wording of the received support scales, partners were asked to assess the emotional support they provided (e.g., "I comforted him/her when he/she was feeling bad"; Cronbach's alpha =.67). Responses were strongly disagree (1), disagree (2), agree (3), strongly agree (4). 


\section{Data Analyses}

Data were analyzed either as Pearson correlations, hierarchical regression analyses, or repeated measures analyses of variance across three points in time (patients) or two points in time (partners), with gender as a between-subjects factor. Missing data (less than $5 \%$, due to omitted responses) were imputed using the SPSS MVA regression procedure.

\section{Results}

\section{Changes in Received Emotional Support of Cancer Patients at Three Points in Time}

It was hypothesized that received emotional support would be higher, in the long run, for male than for female patients. Mean level changes of received emotional support were examined separately for the 108 male and the 65 female patients. Overall, received support levels were very high. Male patients reported the same high level of received emotional support across the six-month study period. In contrast, although women reported the same high levels of received support before surgery and one month after, they reported significantly less emotional support received than male patients six months after surgery. Table 2 displays the means for received emotional support.

A repeated measures analysis of variance with three points in time and with patient gender as a betweensubjects factor was computed. There were main effects for Gender, $F(1,171)=11.64, p<.001, \eta^{2}=.06$, and Time, $F(2,342)=10.14, p<.001, \eta^{2}=.06$, and for the interaction of Time $\mathrm{x}$ Gender, $F(2,342)=$ $8.69, p<.001, \eta^{2}=.05$. Cohen's $d$ for the difference between men and women was $d=0.16$ at Time $1, d=$ 0.36 at Time 2 , and $d=0.68$ at Time 3 .

\section{Changes in Provided Emotional Support by Partners at Two Points in Time}

To examine whether male and female partners provided different amounts of support over time, a re-

Table 2. Spousal Support Received by Patients at Three Points in Time

\begin{tabular}{lccc}
\hline & $\begin{array}{c}\text { Before } \\
\text { Surgery }\end{array}$ & $\begin{array}{c}\text { 1 Month after } \\
\text { Surgery }\end{array}$ & $\begin{array}{c}\text { 6 Months after } \\
\text { Surgery }\end{array}$ \\
\hline Mean & 3.89 & Male patients $(n=108):$ & 3.89 \\
SD & 0.26 & 3.90 & 0.27 \\
& \multicolumn{2}{c}{ Female patients $(n=65):$} \\
Mean & 3.85 & 3.79 & 3.65 \\
SD & 0.28 & 0.38 & 0.42 \\
Mean & 3.87 & Total $(N=173)$ & 3.80 \\
SD & 0.27 & 3.86 & 0.35 \\
\hline
\end{tabular}

Table 3. Support Provided by Partners at Two Points in Time

\begin{tabular}{lcc}
\hline & Before surgery & 1 Month after surgery \\
\hline \multirow{3}{*}{ Mean } & Male partners $(n=65):$ & \\
$S D$ & 3.71 & 3.54 \\
& 0.31 & 0.50 \\
Mean & Female partners $(n=108):$ & \\
$S D$ & 3.77 & 3.74 \\
& 0.34 & 0.32 \\
Mean & Total $(N=173)$ & \\
$S D$ & 3.75 & 3.67 \\
\hline
\end{tabular}

peated measures analysis of variance was computed, with partner gender as the between-groups factor. There were main effects for Gender, $F(1,171)=7.81$, $p<.01, \eta^{2}=.04$, and Time, $F(1,171)=9.33, p<$ $.01, \eta^{2}=.05$, and the Gender $\times$ Time interaction, $F(1$, $171)=4.79, p<.05, \eta^{2}=.03$. Table 3 displays the means for provided emotional support. Overall, provided support levels were very high. Initially, male and female partners provided the same high level of emotional support, but one month after surgery there was a significant decline of support by male caregivers, whereas female partners continued to extend emotional support at the same level as before surgery. This ordinal interaction between Time and partners' Gender underscores the similar pattern that was found previously for received support among patients.

\section{Associations Between Patients' Received Emotional Support and Partners' Provided Emotional Support}

The third research question seeks to understand how support provided by a partner is related to support received by a patient. It was hypothesized that the amount of received support reported by patients would differ from the amount of provided support reported by partners, and that gender differences would emerge. Twelve correlation coefficients, most of them timelagged, were computed separately for men and women (Table 4).

The first row in Table 4 presents the association between support provided by female partners directly before surgery and male patients' received support at three points in time: before surgery, $r=.30, p<$ .05 ; one month after surgery, $r=.20, p<.05$; and six months after surgery, $r=.40, p<.01$. One line below are the correlations of post-surgery provision by women with support received by men at three points in time.

In 4 out of 6 cases support provided by men was associated with support received by female patients. But for the male partners, the picture was slightly different (cf. bottom half of Table 4), $r=.10, p<.05$, 
Table 4. Time-Lagged Dyadic Correlations of Patients' Received Support and Their Partners' Provided Support, Separately for Men $(\mathrm{n}=108)$ and Women $(\mathrm{n}=65)$ Patients

\begin{tabular}{lccc}
\hline \multicolumn{3}{c}{ Patients' } & received emotional support \\
\cline { 2 - 4 } & $\begin{array}{c}\text { Before } \\
\text { surgery }\end{array}$ & $\begin{array}{c}1 \text { Month } \\
\text { after surgery }\end{array}$ & $\begin{array}{c}\text { 6 Months } \\
\text { after surgery }\end{array}$ \\
\hline
\end{tabular}

\begin{tabular}{lccc}
\hline \multicolumn{4}{c}{ Support provided by female partners $(n=108)$ : } \\
Before surgery & $.30^{*}$ & $.20^{*}$ & $.40^{* *}$ \\
1 month after surgery & $.20^{*}$ & $.49^{* *}$ & $.40^{* *}$ \\
\multicolumn{4}{c}{ Support provided by male partners $(n=65):$} \\
Before surgery & .10 & $.29^{*}$ & $.26^{*}$ \\
1 month after surgery & .16 & $.26^{*}$ & $.44^{* *}$ \\
\hline
\end{tabular}

Note: $* p<.05, * * p<.01$.

$r=.29, p<.05, r=.26, p<.05$, respectively. One line below, the correlations of post-surgery provision by men with receipt by women at three points in time are displayed.

Gender emerged as a significant moderator for the provision-receipt relation before surgery, $r=.30$ $>\quad r=.10, Z=1.31, p<.10$, and one month after surgery, $r=.49>\quad r=.26, Z=1.69, p<.05$. That is, within the sample of female providers the association between provision and receipt was closer than in the sample of male providers.

The fourth hypothesis aims at the differential prediction of received support. Hierarchical regression analyses were computed separately for male and female patients, with received support six months after surgery (Time 3 ) as the dependent variable. Time 1 and Time 2 baseline variables (received support) were entered as Block 1, whereas provided support variables were entered as Block 2, making a significant contribution. Results are summarized in Table 5. In the subsample of male patients, the four predictors accounted for $36 \%$ of the variance of Time 3 received support, compared to $58 \%$ in the subsample of female

Table 5. Hierarchical Regression Analysis Predicting Patients' Received Support Half a Year After Surgery (Time 3), Separately for Men $(\mathrm{n}=108)$ and Women $(\mathrm{n}=65)$ Patients

\begin{tabular}{lcc}
\hline Predictors & $\begin{array}{c}\text { Male Patients } \\
\text { Beta }\end{array}$ & $\begin{array}{c}\text { Female patients } \\
\text { Beta }\end{array}$ \\
\hline $\begin{array}{c}\text { Support received by } \\
\text { patients at Time 1 }\end{array}$ & $.29 * *$ & $.30^{*}$ \\
$\begin{array}{l}\text { Support received by } \\
\text { patients at Time 2 }\end{array}$ & $.20^{*}$ & $.40^{* *}$ \\
$\begin{array}{l}\text { Step 1 R } \\
\text { Support provided by } \\
\text { partners at Time 1 }\end{array}$ & $.28^{* *}$ & $.50^{* *}$ \\
$\begin{array}{l}\text { Support provided by } \\
\text { partners at Time 2 }\end{array}$ & $.22^{*}$ & .00 \\
Step 2 $R^{2}$ & .16 & $.29 *$ \\
\hline
\end{tabular}

Note. $* p<.05, * * p<.01$. patients. In particular, the weight of Time 2 support provision by male partners was substantial. Support by male partners (or the lack of it) made a difference, when provided (or not provided) one month after surgery.

\section{Discussion}

This study endeavors to contribute to a better understanding of support provided and support received in couples during times of crisis before and after cancer surgery. In general, members of the 173 dyads reported high levels of support provided and support received, respectively. Men reported receiving more emotional support than women. This pattern remained stable across the entire observation period of half a year. Initially, women experienced the same high levels of support as men did, but six months after surgery they reported less received emotional support than their male counterparts. Being a woman with cancer was associated with receiving a lower amount of spousal support than a man with cancer receives. This is in line with the support-gap hypothesis (Belle, 1982), stating that men receive more from women than vice versa (see also Gurung et al., 2003). Our study added a time perspective to this hypothesis, pointing to a differential development of support received and provided within a half-year period (see also Schwarzer \& GutiérrezDoña, 2005).

Although effects found may look negligible at first glance, when merely comparing the mean levels of men and women, we obtained medium effect sizes, with an interaction of Time $\times$ Gender effect of $5 \%$, and Cohen's $d$ for the difference between men and women of $d=0.16$ at Time $1, d=0.36$ at Time 2 , and $d=0.68$ at Time 3 (i.e., medium effect size). Previous studies revealed that among individuals who were not exposed to any crisis event (i.e., cancer surgery or diagnosis), changes in perceived and received social support are rather small in terms of effect sizes (cf. Pierce et al., 2000). Research on changes in perceived social support among individuals followed for 12 months after a crisis showed Time $\times$ Gender interactions with effect sizes of $4.5 \%$ (Stelle \& Uchida, 2004), similar to the ones obtained in the present study. Consistent with previous research, the obtained effects were not large, but substantial.

It has also been found in other contexts that women receive less spousal support than men (Cutrona, 1996; Glynn et al., 1999; Kunkel \& Burleson, 1999; Pasch \& Bradbury, 1998). Explanations for this may be that (a) men do indeed provide less support to their partners, (b) women do not benefit from support as much as men do, (c) men do not provide support as effectively as women do, or (d) men do not provide effective support in particular to women. On the other hand, women might benefit more from support from female 
friends or relatives (Uno et al., 2002), whereas men seem to benefit emotionally from opposite-sex support (Mickelson et al., 1995). MacGeorge and colleagues (2003) found evidence of a skill deficit in emotional sensitivity in men that might also account for their lower levels of emotional support provision.

The reported emotional support provided by partners corroborates the above findings (Table 3 ). Initially, men and women provided the same high levels of emotional support. However, one month after surgery, there was a significant decline of emotional support by male caregivers, whereas women continued to extend the same amount. This effect mirrors the pattern of received support in patients. A time-delayed effect of declining male partner support might have been manifested half a year later in female patients who reported receiving less emotional support than before.

There is some concordance on the support transaction between men and women (i.e., associations between provided and received support). This can be interpreted as an indication of good relationship functioning. Coriell and Cohen (1995) found that dyad intimacy was associated with greater concordance. This is also in line with research on dyadic support in cancer patients (see Hagedoorn et al., 2000).

Another striking result is the time-delayed effect of initial support provision on later support receipt. The concurrent associations before surgery were lower (.30, .10) than the lagged associations between presurgery provision and received support six months later (.40, .26), in particular the five-month interval from Time 2 to Time $3(.40, .44)$ (see Table 4$)$. Usually, longitudinal correlations are washed out due to various intervening factors. Here, the opposite occurred. Initial associations might have been low if, for example, provided support had not been recognized by the patient. Or, alternatively, support might be more accurately evaluated by patients if it has been provided continuously for several months.

In longitudinal data, subsequent self-reports are often determined by previous self-reports. To control for the baseline reporting of received support, we have computed a hierarchical regression analysis with the baseline as the first step and then examined whether provision of support would account for more variance beyond the baseline. There was a significant contribution of the provision variables of $8 \%$ (in both men and women). In particular, Time 2 support by male partners was substantial. Their support (or the lack of it) made a difference when provided (or not provided) one month after surgery (see Table 5).

Although this study has its strengths in its longitudinal design, sample size, and dyadic perspective, there are also limitations. First, self-reports fail to fully capture the actual exchange of support behaviors between spouses. Future research should include observation methods to analyze what is occurring in couples during times of stress and conflict (Pasch \& Bradbury, 1998). Also, dyadic characteristics, such as length and quality of the relationship, marital history, conflicts, and patterns of relations between provided and received support for patients with other diseases after obtaining a diagnosis or aggressive treatment should be more closely examined in the future.

Second, provided and received support have not been assessed in a reciprocal manner. There are no data on received support in partners or on support provided by patients with cancer. Such dyadic data have been collected in couples with infertility, for example, where similarities in support received and provided were assessed, as well as agreement between husbands and wives (Abbey et al., 1995). To continue to better understand dyadic coping and support transactions, more needs to be known about mutual provided and received support, as well as conflict and tension among couples. Reciprocity is seen as one determinant of support provision (Cutrona et al., 1997; Gleason et al., 2003; Jung, 1990; Knoll et al., 2006a; Knoll et al., 2006b; Liang et al., 2001). Therefore, it would be valuable to obtain data that allow for a closer examination of such reciprocal effects (Kenny \& Cook, 1999; Newsom, 2002).

Third, the choice of time windows is an unresolved issue in this kind of field research on social support in times of crisis. The study was designed in line with other studies, choosing time points before and after surgery as well as at follow-up. Support is an ongoing process, but selection of the appropriate time windows for assessment remains an arbitrary decision. If, for example, patients report having received support during the last week at half a year post surgery, then it remains unknown whether the statements refer only to the last week, or whether they are also applicable to the entire last five months when the previous assessment took place. In other words, it is unclear for which time period the partner effect is strongest. In the female patient/male partner dyad, there was no substantial cross-sectional relation between provision and receipt, but there were delayed effects extending over one month and five months. We do not know whether the strongest effect had occurred after a few days or after a couple of weeks. Emotional support does not constitute a single act, but it might be expressed by daily hospital visits or daily care at home. Therefore, more data on the interactions within couples at multiple time windows are needed. Moreover, the time window of six months may be too short to see how differently support by men and by women may eventually operate in the long run. As another limitation, the present data did not include further potential moderators, such as financial concerns and other life stressors that might have impacted the support.

Fourth, the wording of support items did not exclude nonspouses. Patients were asked to identify their closest significant other and to give that person 
the partner questionnaire. Of all significant others, 173 were intimate partners; these couples constituted the present sample. The measurement of received support, however, was not explicit for intimate partners because the wording had to match all significant others who were included in the study. Thus, it could be possible that a married patient might have thought of a different close support provider than his/her spouse when responding to the support items. This is, however, unlikely because identification of the target person for the partner questionnaire and identification of the closest support person occurred within the same hour of data collection, and only couples were included in this analysis. If a patient would, for example, regard his child (instead of his spouse) as the prime support provider, then he should also have assigned his child as the recipient for the questionnaire. The procedure used in data collection ensured that all data collected referred to the same close person.

The results also have clinical implications. Medical personnel should be aware that female patients with cancer may not be receiving as much continuous support from their partners as their male counterparts. Thus, two implications might be considered. First, patients need help to think of multiple ways they can get the support they require or to think about what they could do if they are not getting such support from their partner, acknowledging that support provided from partners can change over time. Alternative sources of support, such from friends or professional help, may need to be identified. Second, a couple-coping intervention (Scott et al., 2004) may be implemented to help partners learn about each other's needs in times of crisis and ways to cope with difficulties after cancer surgery.

\section{References}

Abbey, A., Andrews, F. M., \& Halman, L. J. (1995). Provision and receipt of social support and disregard: What is their impact on the marital life quality of infertile and fertile couples? Journal of Personality and Social Psychology, 68, 455-469.

Belle, D. (1982). The stress of caring: Women as providers of social support. In L. Goldberger \& S. Breznitz (Eds.), Handbook of stress: Theoretical and clinical aspects (pp. 496-505). New York: Free Press.

Bodenmann, G. (1997). Dyadic coping-a systemic-transactional view of stress and coping among couples: Theory and empirical findings. European Review of Applied Psychology, 47, 137-140.

Bolger, N., Zuckerman, A., \& Kessler, R. C. (2000). Invisible support and adjustment to stress. Journal of Personality and Social Psychology, 79, 953-961.

Burleson, B. R. (2003). The experience and effects of emotional support: What the study of cultural and gender differences can tell us about close relationships, emotion, and interpersonal communication. Personal Relationships, 10, 1-23.

Clark, S. L., \& Stephens, M. A. P. (1996). Stroke patients' wellbeing as a function of caregiving spouses' helpful and unhelpful actions. Personal Relationships, 3, 171-184.

Coriell, M., \& Cohen, S. (1995). Concordance in the face of a stressful event: When do members of a dyad agree that one person supported the other? Journal of Personality and Social Psychology, 69, 289-299.

Coyne, J. C., \& Fiske, V. (1992). Couples coping with chronic illness. In T. J. Akamatsu, J. C. Crowther, S. C. Hobfoll, \& M. A. P. Stephens (Eds.), Family health psychology (pp. 129-149). Washington, DC: Hemisphere.

Coyne, J. C., \& Smith, D. A. F. (1991). Couples coping with a myocardial infarction: A contextual perspective on wives' distress. Journal of Personality and Social Psychology, 61, 404412.

Cutrona, C. E. (1996). Social support in couples. Thousand Oaks, CA: Sage.

Cutrona, C. E., Hessling, R. M., \& Suhr, J. A. (1997). The influence of husbands and wife personality on marital social support interactions. Personal Relationships, 4, 379-393.

DeLongis, A., Capreol, M. J., Holtzman, S., O'Brien, T. B., \& Campbell, J. (2004). Social support and social strain among husbands and wives: A multilevel analysis. Journal of Family Psychology, $18,470-479$.

Gleason, M. E. J., Iida, M., Bolger, N., \& Shrout, P. E. (2003). Daily supportive equity in close relationships. Personality and Social Psychology Bulletin, 29, 1036-1045.

Glynn, L. M., Christenfeld, N., \& Gerin, W. (1999). Gender, social support, and cardiovascular responses to stress. Psychosomatic Medicine, 61, 234-242.

Goldsmith, D. J., \& Dun, S. A. (1997). Sex differences and similarities in the communication of social support. Journal of Social and Personal Relationships, 14, 317-337.

Gurung, R. A. R., Taylor, S. E., \& Seeman, T. E. (2003). Accounting for changes in social support among married older adults: Insights from the MacArthur studies on successful aging. Psychology and Aging, 18, 487-496.

Hagedoorn, M., Kuijer, R. G., Buunk, B. P., De Jong, G. M., Wobbes, T., \& Sanderman, R. (2000). Martial satisfaction in patients with cancer: Does support from intimate partners benefit those who need it the most? Health Psychology, 19, 274 282.

Helgeson, V. S. (1993). The onset of chronic illness: Its effect on the patient-spouse relationship. Journal of Social and Clinical Psychology, 12, 406-428.

Jung, J. (1990). The role of reciprocity in social support. Basic and Applied Social Psychology, 11, 243-253.

Kenny, D. A., \& Cook, W. (1999). Partner effects in relationship research: Conceptual issues, analytic difficulties, and illustrations. Personal Relationships, 6, 433-448.

Kiecolt-Glaser, J. K., McGuire, L., Robles, T. F., \& Glaser, R. (2002). Psychoneuroimmunology and psychosomatic medicine: Back to the future. Psychosomatic Medicine, 64, 15-28.

Klauer, T., \& Winkeler, M. (2002). Gender, mental health status, and social support during a stressful event. In G. Weidner, M. Kopp, \& M. Kristenson (Eds.), Heart disease: Environment, stress, and gender. NATO Science Series, Series I: Life and Behavioural Sciences, Vol.327 (pp. 223-236). Amsterdam: IOS Press.

Knoll, N., Burkert, S., \& Schwarzer, R. (2006a). Reciprocal support provision: Personality as a moderator? European Journal of Personality, 20, 217-236.

Knoll, N., Schulz, U., Schwarzer, R., \& Rosemeier, H. P. (2006b). Provider's appraisal detection bias and the efficacy of received support in medical students preparing for an exam. British Journal of Social Psychology, 45, 599-615.

Kunkel, A. W., \& Burleson, B. R. (1999). Assessing explanations for sex differences in emotional support: A test of the different cultures and skill specialization accounts. Human Communication Research, 25, 307-340.

Liang, J., Krause, N. M., \& Bennett, J. M. (2001). Social exchange and well-being: Is giving better than receiving? Psychology and Aging, 16, 511-523. 
MacGeorge, E. L., Gillihan, S. J., Samter, W., \& Clark, R. A. (2003). Skill deficit or differential motivation? Testing alternative explanations for gender differences in the provision of emotional support. Communication Research, 30, 272-303.

Manne, S. L., Pape, St. J., Taylor, K. L., \& Dougherty, J. (1999). Spouse support, coping, and mood among individuals with cancer. Annals of Behavioral Medicine, 21, 111-121.

Mickelson, K. D., Helgeson, V. S., \& Weiner, E. (1995). Gender effects on social support provision and receipt. Personal Relationships, 2, 211-224.

Moyer, A., \& Salovey, P. (1999). Predictors of social support and psychological distress in women with breast cancer.Journal of Health Psychology, 4, 177-191.

Neff, L. A., \& Karney, B. R., (2005). Gender differences in social support: A question of skill or responsiveness? Journal of Personality and Social Psychology, 88, 79-90.

Newsom, J. T. (2002). A multilevel structural equation model for dyadic data. Structural Equation Modeling, 9, 431-447.

Northouse, L. L., Mood, D., Templin, T., Mellon, S., \& George, T. (2000). Couples' patterns of adjustment to colon cancer. Social Science and Medicine, 50, 271-284.

Pasch, L. A., \& Bradbury, T. N. (1998). Social support, conflict, and the development of marital dysfunction. Journal of Consulting and Clinical Psychology, 66, 219-230.

Pierce, R. S., Frone, M. R., Russell, M., Cooper, M. L., \& Mudar, P. (2000). A longitudinal model of social contacts, social support, depression, and alcohol use. Health Psychology, 19, 28-38.

Revenson, T. A. (1994). Social support and marital coping in chronic illness. Annals of Behavioral Medicine, 6, 122-130.

Revenson, T. A., Kayser, K., \& Bodenmann, G. (Eds.). (2005). Couples coping with stress: Emerging perspectives on dyadic coping. Washington, DC: American Psychological Association.
Schulz, U., \& Schwarzer, R. (2003). Soziale Unterstützung bei der Krankheitsbewältigung: Die Berliner Social Support Skalen (BSSS) [Social support and coping with illness: The Berlin Social Support Scales [BSSS]). Diagnostica, 49, 73-82.

Schulz, U., \& Schwarzer, R. (2004). Long-term effects of spousal support on coping with cancer after surgery. Journal of Social and Clinical Psychology, 23, 716-732.

Schwarzer, R., Dunkel-Schetter, C., \& Kemeny, M. (1994). The multidimensional nature of received social support in gay men at risk of HIV infection and AIDS. American Journal of Cоттиnity Psychology, 22, 319-339.

Schwarzer, R., \& Gutiérrez-Doña, B. (2005). More spousal support for men than for women: A comparison of sources and types of support. Sex Roles: A Journal of Research, 52, 523-532.

Schwarzer, R., Knoll, N., \& Rieckmann, N. (2004). Social support. In A. Kaptein \& J. Weinman (Eds.), Health Psychology (pp. 158-182). Oxford, England: Blackwell.

Scott, J. L., Halford, W. K., \& Ward, B. G. (2004). United we stand? The effects of a couple-coping intervention on adjustment to early stage breast or gynecological cancer. Journal of Consulting and Clinical Psychology, 72, 1122-1135.

Stelle, C. D., \& Uchida, M. (2004). The stability and change in the social support networks of widowers following spousal bereavement. Journal of Men's Studies, 13, 85-105.

Turner-Cobb, J. M., Sephton, S. E., Koopman, C., Blake-Mortimer, J., \& Spiegel, D. (2000). Social support and salivary cortisol in women with metastatic breast cancer. Psychosomatic Medicine, $62,337-345$.

Uno, D., Uchino, B. N., \& Smith, T. W. (2002). Relationship quality moderates the effect of social support given by close friends of cardiovascular reactivity in women. International Journal of Behavioral Medicine, 9, 243-262. 
Copyright of International Journal of Behavioral Medicine is the property of Lawrence Erlbaum Associates and its content may not be copied or emailed to multiple sites or posted to a listserv without the copyright holder's express written permission. However, users may print, download, or email articles for individual use. 